ESAIM: PROCEEDINGS, October 2007, Vol. 22, 108-113

Gabriel Caloz \& Monique Dauge, Editors

\title{
ELECTROMAGNETIC MODELLING OF RADIOWAVES AND HUMAN BODIES INTERACTIONS
}

\author{
MAN-FAÏ WONG ${ }^{1}$ AND JOE WiarT ${ }^{1}$
}

\begin{abstract}
Electromagnetic modelling has become essential in the assessment of human exposure to radiowaves. The ever growing widespread use of wireless technologies around human bodies brings new studies for compliance analyses or exposure systems designs for biomedical researches. Numerical techniques have to be adapted to derive efficient models to tackle the complexity of phenomena. Recent developments are presented on the interactions with terminals or base station antennas: comparison of absorption between child and adult heads, source body coupling, accurate antenna modelling.
\end{abstract}

Résumé. La modélisation électromagnétique est devenue indispensable dans l'évaluation des interactions des ondes radiofréquences avec les personnes. L'utilisation de plus en plus intensive et proche des personnes des technologies sans fil ouvre une grande variété de situations à analyser dans les études de conformité ou d'élaboration de systèmes d'exposition destinés aux études biomédicales. Pour cela, les modèles et leurs utilisations doivent être adaptés pour gérer la complexité des phénomènes. Nous présentons dans ce papier quelques développements récents répondant à des problématiques actuelles aussi bien en ce qui concerne les terminaux que les antennes des stations de base : comparaison de l'exposition des enfants par rapport aux adultes, analyse du couplage source corps humain, développement de modèles d'antennes précis.

\section{INTRODUCTION}

Solving Maxwell equations using numerous numerical techniques, Finite Element Methods, Finite Differences, Finite Volumes, integral equation based methods has become essential for engineering problems involving electromagnetic waves. The application of the numerical techniques in bioelectromagnetics allows the dosimetry of exposure configurations involving an electromagnetic source and a human body: compliance studies where the field levels are compared to the exposure limits, understanding and quantifying the wave tissue interactions for the biomedical studies [1]. The modelling of human bodies is very challenging as mesh based techniques have to deal with millions of elements involved by the highly heterogeneous media of human bodies. The Yee scheme based Finite Difference Time Domain (FDTD) is a simple and powerful method for solving many wave engineering problems [2]. The FDTD is the most popular method for modelling electromagnetic interactions with human bodies as the voxels of 3D segmented MRI (Magnetic Resonance Imaging) images of biological tissues are simply the cells of the FDTD grid. The explicit time scheme of the FDTD avoids the large system solving found in unstructured techniques. This brute force and fullwave technique is very successful but has also some limitations with the ever increasing complexity of the real life situations. The intrinsic staircasing of arbitrary geometries in FDTD drives improvements of the standard algorithm like conformal schemes. Higher frequencies of new wireless technologies or higher resolution asked by microdosimetry, or simply adaptive resolution calls

\footnotetext{
${ }^{1}$ France Telecom Research \& Development, 38-40 rue du Général Leclerc, 92794 Issy Moulineaux, France;

e-mail: manfai.wong@orange-ftgroup.com \& joe.wiart@orange-ftgroup.com
}

(c) EDP Sciences, SMAI 2007 

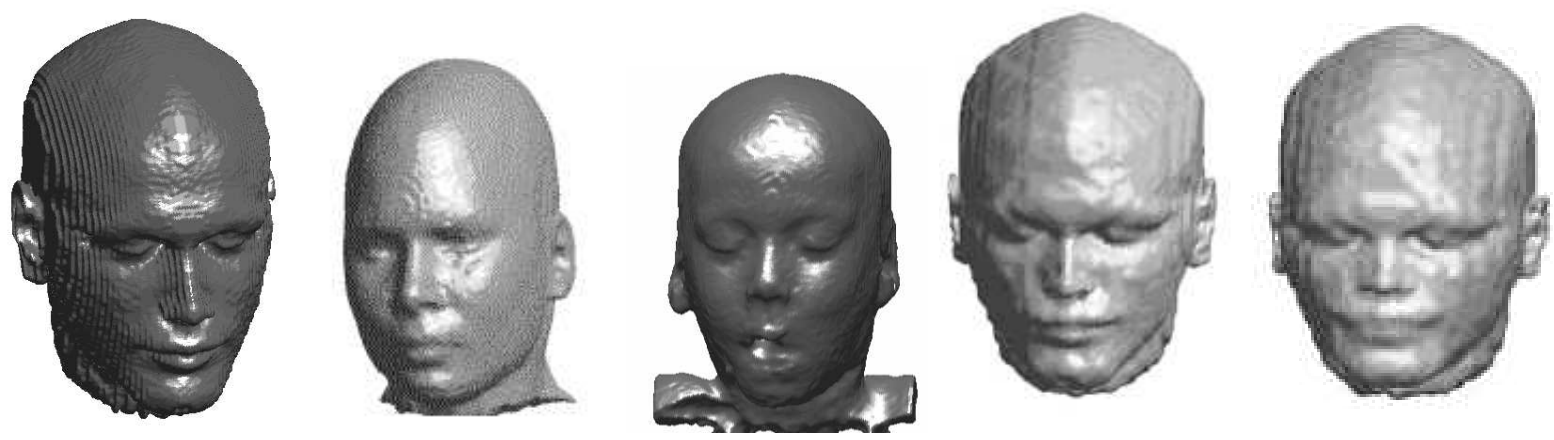

Figure 1. Head models (from left to right): adult, 12 years old child, 4 years old child, CL 12 years old derived from adult, CL 4 years old derived from adult

for graded mesh or subgridding techniques to overcome the uniform grids of the Yee scheme. Hybridation with other techniques have also been investigated to improve the efficiency. Fast uncertainty or sensitivity analyses due to geometrical or dielectric parameters are also of interest. This is essential to evaluate the representativeness of an analysed configuration. Three recent developments will illustrate the needs in numerical modelling. They are related to the mobile phones and the base station antennas which are the two systems of exposure interest in a mobile network. The way the source and the body is coupled is also discussed giving adaptive and efficient techniques.

\section{SAR INDUCED IN CHILD HEAD}

Mobile phones are so familiar in our every day life, their use by children is increasing. Questions are raised on the specifity of the exposure of children head compared to adult one. Is the absorption of electromagnetic waves greater in child head due to the different morphology or dielectric properties ? In the framework of RNRT project ADONIS [3], the Specific Absorption Rate (SAR) induced in child head by mobile terminals has been analysed. Morphology of child head and its evolution with respect to the age are to be taken into account in the development of head models. Representativeness of models is of prime importance. The simplest models consist in down scaling adult head models. Clearly they are crude models of child head. A better technique is to make an anisotropic scaling with respect to some external parameters. These kind of models are named "Child Like" (CL). From an adult head, different child head corresponding to different ages are easily obtained. However, this morphing transformation does not ensure correct reconstruction of internal tissues. Therefore, real child head models have been obtained from MRI images in the ADONIS project. The FDTD is used to calculate the fields: handset close to the head is routinely analysed. Interesting comparisons can then be made between the SAR induced in the different head models to evaluate the uncertainty linked to their representativeness of real heads [4].

Here, 3 head models derived from MRI are used: adult, 12 years old child and 4 years old child. Two CL models, 4 and 12 years old, are also considered (Fig.1). These millimeter resolution head models are exposed to 2 mobile phone models: a triband mobile (900 MHz, $1800 \mathrm{MHz}$ and $2100 \mathrm{MHz}$ ) (Fig.2, left) and the IEEE monopole on a box working at $835 \mathrm{MHz}$ and $1900 \mathrm{MHz}$ (Fig.2, right

). Table 1 shows the proportion of absorbed power in the different configurations. At $900 \mathrm{MHz}$ and 835 $\mathrm{MHz}$, about $2 / 3$ of emitted power is absorbed while it is $1 / 3$ at higher frequencies $(1800 \mathrm{MHz}, 1900$ and 2100 $\mathrm{MHz}$ ). The absorbed power results for the child heads are comparable to those for the adult head. Figure 3 (left) shows the peak local SAR in $10 \mathrm{~g}$. The peak SAR is higher for the adult head than for the child ones at all frequencies except at $900 \mathrm{MHz}$ where the 4 years old CL gives the highest value. The SAR in $1 \mathrm{~g}$ relative to the tissues are compared in Fig.3 (right) at $900 \mathrm{MHz}$. Highest values are obtained for the skin. In these cases, CL models give higher values than MRI models. 

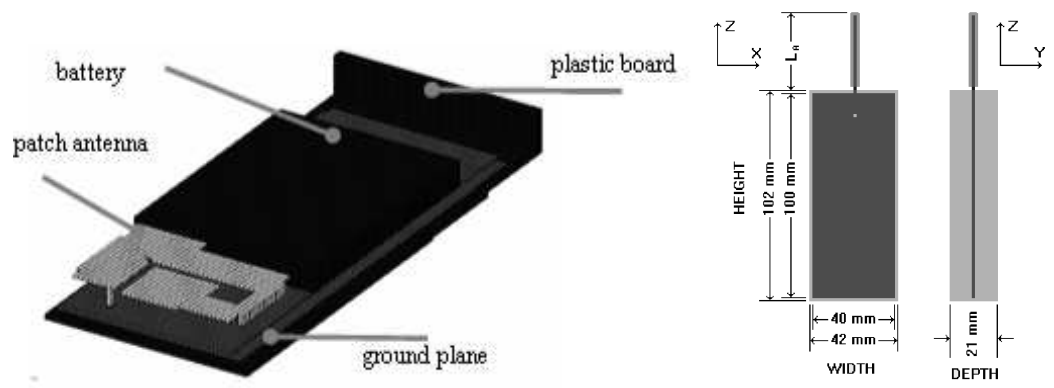

Figure 2. Triband mobilephone model: internal components (left), IEEE monopole (right)

\begin{tabular}{|c|c|c|c|c|c|}
\hline Frequency $(\mathrm{MHz})$ & Adult & CL 12 yo & Child 12 yo & CL 4 yo & Child 4 yo \\
\hline 900 & $58 \%$ & $59 \%$ & $53 \%$ & $55 \%$ & $58 \%$ \\
1800 & $32 \%$ & $34 \%$ & $32 \%$ & $28 \%$ & $36 \%$ \\
2100 & $42 \%$ & $45 \%$ & $40 \%$ & $42 \%$ & $46 \%$ \\
\hline 835 & $55 \%$ & $51 \%$ & $60 \%$ & $53 \%$ & $60 \%$ \\
1900 & $35 \%$ & $37 \%$ & $44 \%$ & $30 \%$ & $50 \%$ \\
\hline
\end{tabular}

TABLE 1. Percentage of absorbed power
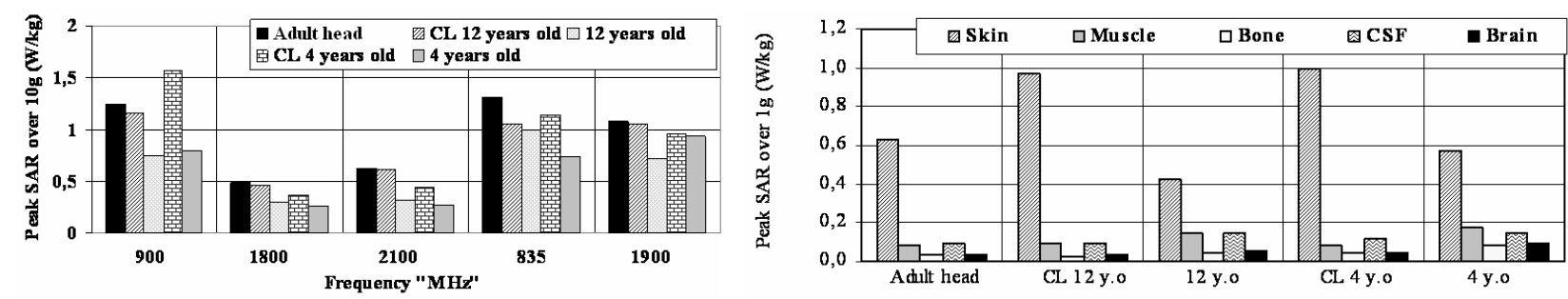

Figure 3. SAR $10 \mathrm{~g}$ vs. frequency (left). SAR $1 \mathrm{~g}$ in tissues at $900 \mathrm{MHz}$ (right).

\section{SAR AND COUPLING OF BASE STATION ANTENNAS}

Another typical situation that can be analysed by FDTD is the case of base station antennas that form with the mobile phone the two ends of the radiolink. For the safety of workers or people approaching these antennas, the absorption of electromagnetic waves by human bodies has to be evaluated. The comparison of the whole body SAR and the local SAR with the exposure limits [5] allows to define compliance boundaries outside which the exposure is below the limits. The simulation of the complete configuration source and body, specially in case of millimeter resolution, may require excessive computer resources. If the distance between the source and the body is small, strong coupling exists and a full-wave approach is needed (Fig.4, left). But when the distance becomes greater, one may wonder if a one way coupling can be sufficient which is the usual approach when the distance is infinite. The FDTD as a volumetric technique will need to mesh the space in between which is inefficient and adds numerical dispersion error through the propagation of waves in between. Thus, an hybrid technique coupling the FDTD to deal with the body and another technique for the source, e.g. an integral equation technique like those implemented in FEKO [6], is more efficient. Furthermore, the coupling can be simplified as a one way coupling from the source to the body. The incident field is evaluated in a first step then it is applied on the Huygens box in the FDTD grid (Fig.4, right) in a second step. As an example, the Zubal human body [7] is exposed to a base station antenna working at $2100 \mathrm{MHz}$ characterised with FEKO 

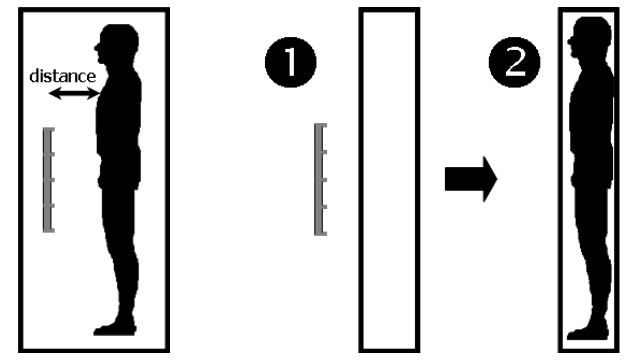

Figure 4. Antenna body coupling: full (left), one way coupling (right)
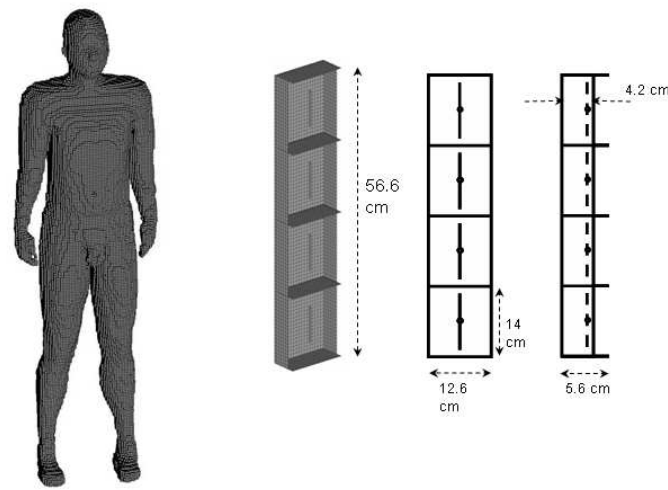

FiguRE 5. Human body model and base station antenna model
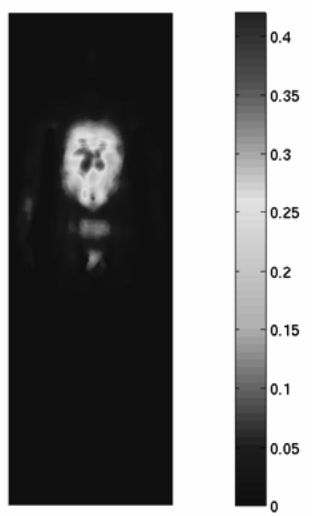

FDTD
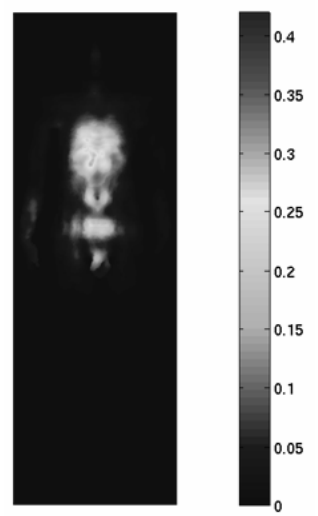

$F E K O+F D T D$

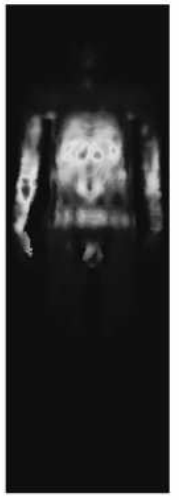

FDTD

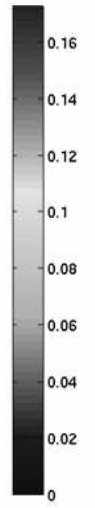

FEKO + FDTD

FIGURE 6. SAR patterns differences: $15 \mathrm{~cm}$ distance (top), $45 \mathrm{~cm}$ distance (bottom)

(Fig.5). The distance is varied from $5 \mathrm{~cm}$ to $90 \mathrm{~cm}$ and the results for the FDTD alone and the FEKO+FDTD are compared. In Fig.6, at $15 \mathrm{~cm}$ some differences on the SAR distribution can be noticed while at $45 \mathrm{~cm}$ they are smaller. The differences for the whole body SAR and the local SAR with respect to the distance are shown in Fig.7: beyond $20 \mathrm{~cm}$ for the whole body SAR, there is less than $5 \%$ difference between the 2 techniques; difference is less than $5 \%$ for the local SAR when the distance is higher than $40 \mathrm{~cm}$. 

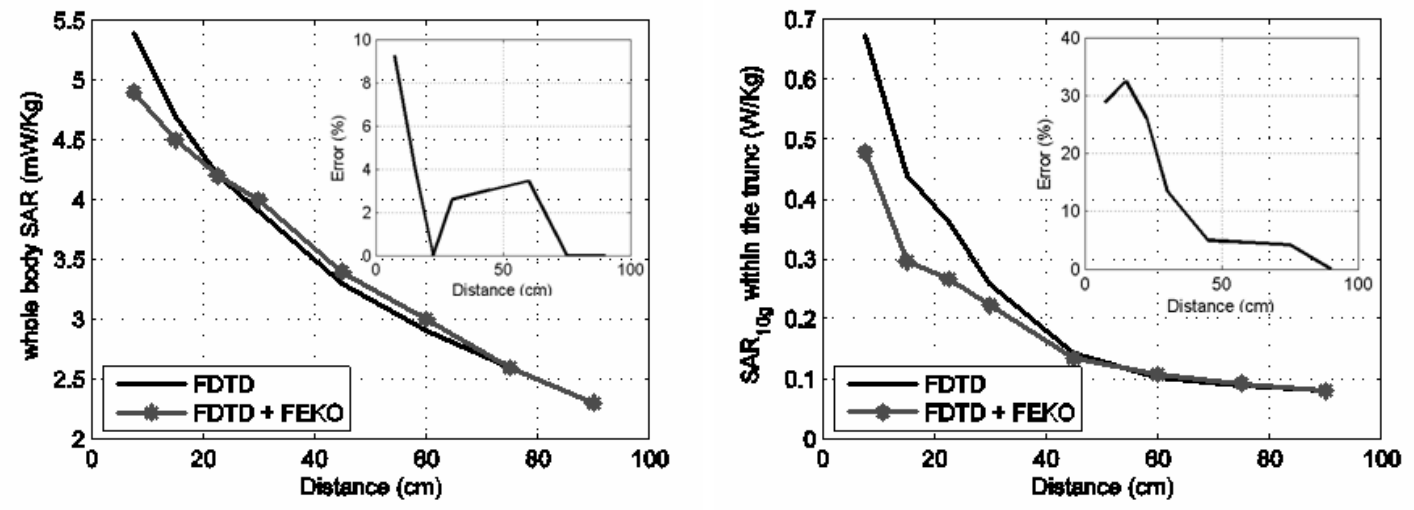

FiguRE 7. SAR comparisons: whole body (left), local (right)

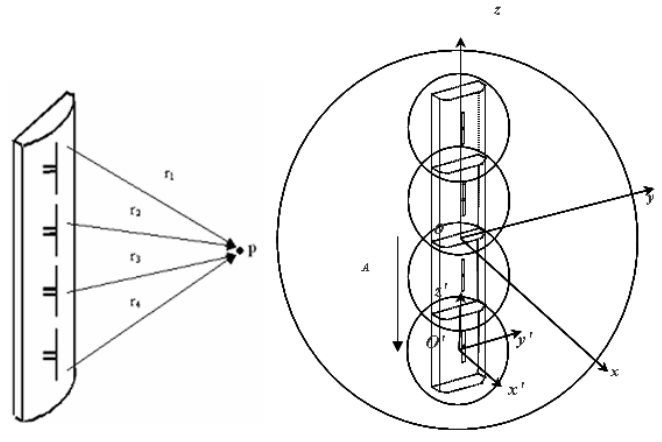

FiguRE 8. Base station antenna decomposed in subantennas

\section{INVERSE MODELLING OF BASE STATION ANTENNAS}

In the one way coupling technique, the incident field provided by the source can also be obtained through measurement. This kind of hybridation is very interesting from an engineering perspective. It may be difficult to model a real antenna in numerical method by giving its geometrical and electrical characteristics. Commercially available antenna can be measured, i.e. the gain and the radiation pattern in free space can be determined. But how to apply the far field data provided by the radiation pattern in the near field region as the region of interest is generally close to the antenna? The synthesis of source models by decomposing the antennas into subantennas characterised by their radiation patterns is a way to answer the question (Fig.8) [8]. The technique has been further improved using spherical harmonics to characterise the subantennas, they are the unknowns in an inverse problem [9] provided that a 3D radiation pattern is given. Fig.9 compares the electric field strength from the measurement and the synthesized model. The agreement is very good, less than $5 \%$. This technique enables the determination of accurate compliance boundaries using electric field strength in the near field region as shown in Fig.9 where the field is reconstructed inside the measurement sphere. These kind of source models can be coupled to the FDTD but also to ray tracing techniques to evaluate the human exposure in urban environment. 

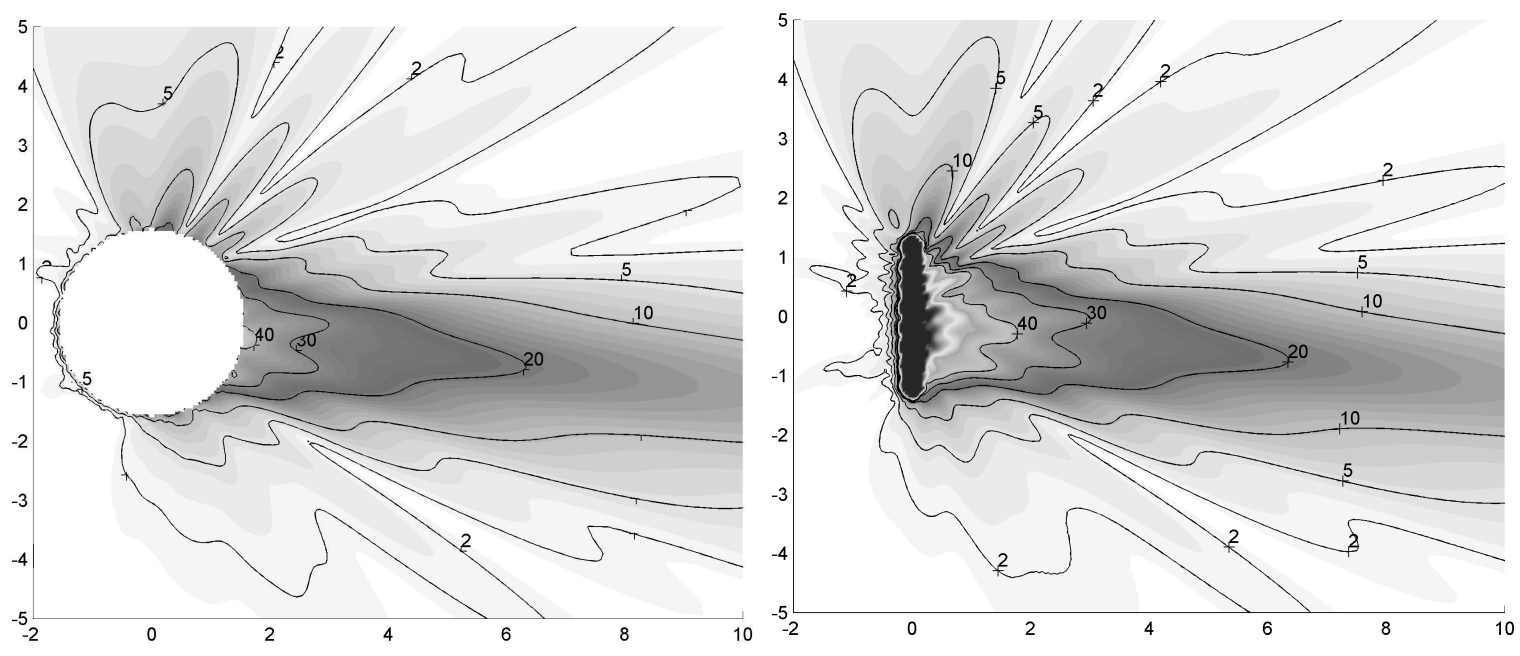

FIGURE 9. Electric field strength: measurement (left), model (right)

\section{Conclusion}

Electromagnetic numerical modelling applied to engineering problems of human exposure to radiowaves has been presented. The FDTD is able to deal with high resolution human body models. Depending on the type of coupling or interaction (close near field, near field or far field), it can be hybridised to improve its efficiency. Measured sources can also be coupled via inverse modelling making simulations more practical or realistic. Existing and emerging wireless technologies bring ever growing complexity real life problems to be analysed. The FDTD has to be adapted with new features like efficient and stable subgridding techniques, hybrid schemes including multiphysics. New techniques like higher order spectral or discontinuous Galerkin techniques need to be further developed. Uncertainty, sensitivity or variability analyses are also of high interest to draw conclusions from simulations.

\section{REFERENCES}

[1] M. F. Wong and J. Wiart, "Modelling of electromagnetic waves interactions with human bodies," C. R. Académie des Sciences, Physique, vol. 6, no. 6, pp. 585-594, 2005.

[2] A. Taflove and S. C. Hagness, Computational Electrodynamics: The Finite-Difference Time-Domain Method, 3rd ed. Norwood, MA: Artech House, 2005.

[3] RNRT Adonis project, "http://www.tsi.enst.fr/adonis/"

[4] J. Wiart, A. Hadjem, N. Gadi, I. Bloch, M. F. Wong, A. Pradier, D. Lautru, V. Fouad Hanna, and C. Dale, "Modeling of RF head exposure in children," Bioelectromagnetics, vol. 26, no. S7, pp. S19-S30, 2005.

[5] ICNIRP, "Guidelines for limiting exposure to time-varying electric, magnetic, and electromagnetic fields (up to $300 \mathrm{GHz}$ )," Health Physics, vol. 74, n4, April 1998.

[6] FEKO, http://www.feko.info/

[7] Zubal phantom, http://noodle.med.yale.edu/zubal/index.htm

[8] Z. Altman, B. Bégasse, C. Dale, J. Wiart, A. Karwowski, M. F. Wong, and L. Gattoufi, "Efficient base station antenna models for dosimetric analysis," IEEE Trans. Electromagnetic Compatibility, vol. 44, pp. 588-592, November 2002.

[9] Y. Adane, A. Gati, M.-F. Wong, C. Dale, J. Wiart, and V. F. Hanna, "Optimal modeling of real radio base station antennas for human exposure assessment using spherical-mode decomposition," IEEE Antennas and Wireless Propagation letters, vol. 1, pp. 215-218, 2003. 